\title{
Lithium Batteries Cooling by Phase Change Material Partially Filled with Metal Foam
}

\author{
Bernardo Buonomo, Fabio d'Alesio, Oronzio Manca, Ferdinando Menale, Sergio Nardini \\ ${ }^{1}$ Università degli Studi della Campania “Luigi Vanvitelli”, Dipartimento di Ingegneria, Via Roma 29, \\ 81031 (Aversa, Italy)
}

\begin{abstract}
Electric cars can be a turning point for climate problems. One of the main problems of electric cars is the thermal control of the batteries, since below and above a certain temperature range, the vehicle's range decreases abruptly, creating inconveniences to the owners of these cars. The thermal control of lithium batteries for electric cars must take into account both the problems of thermal rise due to the operation of the battery itself, and the climatic conditions outside the vehicle that negatively affect the performance of the car, reducing both the autonomy and the battery life. In this study, a thermal control system based on a phase change material (PCM) partially filled with metallic foam is investigated to evaluate its possible use in the cooling of lithium batteries. A two-dimensional model is considered to numerically study thermal control with different chargedischarge cycles. The metal foam partially fills the PCM. The governing equations, written assuming the local thermal equilibrium for the metal foam, are solved by the finite volume method using the ANSYS Fluent commercial code. Different cases are simulated for different values of the external convective heat transfer coefficient. The results, carried out for metal foams and PCM, are given in terms of temperature and liquid fraction. In addition, some comparisons with pure PCM and fully foam filled PCM are provided within the thermal control system to show the advantages of the composite thermal control system with PCM inside the metal foam.
\end{abstract}

\section{Introduction}

In recent years, electric cars have been enjoying great success both among manufacturers, who are strongly encouraging their production and technological development, and among consumers. The reasons that are pushing towards this technology are mainly due to the serious environmental problems due to the pollutants of internal combustion engines and, therefore, to the growing awareness of governments with strong incentive policies for renewable resources in order to give a future of sustainability to this planet. One of the main solutions adopted to limit environmental pollution is to encourage the use of electric cars in residential areas and this, in addition to being an important factor for the livability of urban areas, also represents an economic development factor linked to green economy.

Electric cars use, instead of classic internal combustion engines, an electric motor, which uses chemical energy as primary energy that is stored through rechargeable batteries and then made available to the engine as electrical energy. Electric vehicles are more energy efficient than internal combustion vehicles, but they have some problems due to the use of batteries. These vehicles use lithium-ion batteries, which require long recharging times for low ranges 
and have severe thermal problems that can make their use dangerous. Research on the thermal control of this type of battery is essential to avoid both risks to human health and economic damage to manufacturing companies. Over the years, many techniques have been developed for the thermal control of these devices and one of the most promising and current is the combined use of phase change materials (PCM) and metal foams.

Studies for the application of phase change materials (PCM) and metal foams for the battery management system were performed by Libeer et al [1]. They reported that the use of metal foam with phase change materials improved the heat transfer of the devices they analyse. The optimal temperature range for the use of these devices was studied by Landini et al. [2]. They reported that the typical temperature range for battery operation is $25-40^{\circ} \mathrm{C}$, while the optimum range for battery operation is $25-30^{\circ} \mathrm{C}$. As shown by the studies by Lafdi et al. [3] concerning metal foams with PCMs, the larger pores of the foam give lower surface temperatures due to the high convective flow. Studies conducted by Safdari et al. [4] showed that the best shape to give for thermal management of batteries, among the different geometric shapes from his group studied, is the rectangular shape. Research carried out by Buonomo et al. $[5,6]$ showed that the high latent heat value of PCM allows to delay the reaching of the critical battery temperature until the completion of liquefaction and this, combined with the use of metal foams, allowed for a uniform distribution of the thermal energy in the PCM avoiding that it, given its low thermal conductivity, acts as a thermal insulator.

Research on the thermal response of lithium batteries at high discharge rates through the use of a new Thermal Management System (TMS) that allows for the combination of active and passive methods was conducted by Mashayekhi et al. [7]. They showed that the passive cooling was inefficient in keeping the battery temperature below the safe limit at high discharge rates, while the active hybrid system showed adequate thermal performance under the same conditions. A numerical study of heat transfer in a lithium-ion battery casing using copper metal foams and paraffin phase change material was conducted by Veismoradi et al. [8]. They found that for high heat pulse powers, the melt volume fraction (MVF) increases and the heat sink will have higher efficiency. For a relatively strong heat pulse, the efficiency was improved by about seven times. A compound of metal foams and paraffin phase change material by the vacuum impregnation method was performed by El Idi et al [9]. They modeled the thermal conductivity of the foam and paraffin composite as a function of the structure of the foam, the thermal conductivity of the metal foam and the thermal conductivity of the PCM. The obtained results show a high impact of the thermal conductivity of the metal foam and a smaller effect of the pore size on the effective thermal conductivity.

In the literature there are still rare works on the combination of metal foams with Phase Change Materials (PCM) for the thermal control of lithium-ion batteries, in particular with regards to the use of PCM partially filled with metal foams. This study investigates the promising combination of PCM with metal foams. The paper is a new and original work for thermal control of lithium batteries, which can increase the safety and efficiency of these devices.

\section{Physical model and governing equations}

The physical domain consists of an enclosure filled by a paraffin PCM, RT42, and a copper foam 40 PPI (Pores Per Inch) with a porosity equal to 0.934 . The thickness of the metal foam, $\mathrm{H}_{\mathrm{F}}$, can assume different values.

The operating temperature of the lithium batteries is expected to remain around $318 \mathrm{~K}$ [11]. 
Figure 1 shows the 2D domain used in this study where the dimensions, obtained from a commercial electric battery, are: $\mathrm{L}=310 \mathrm{~mm}, \mathrm{H}=40 \mathrm{~mm}$, for $\mathrm{PCM}$ with Foam $\mathrm{H}_{\mathrm{F}}=1 / 4 \mathrm{H}, 1 / 2$ $\mathrm{H}, 3 / 4 \mathrm{H}, \mathrm{H}$.

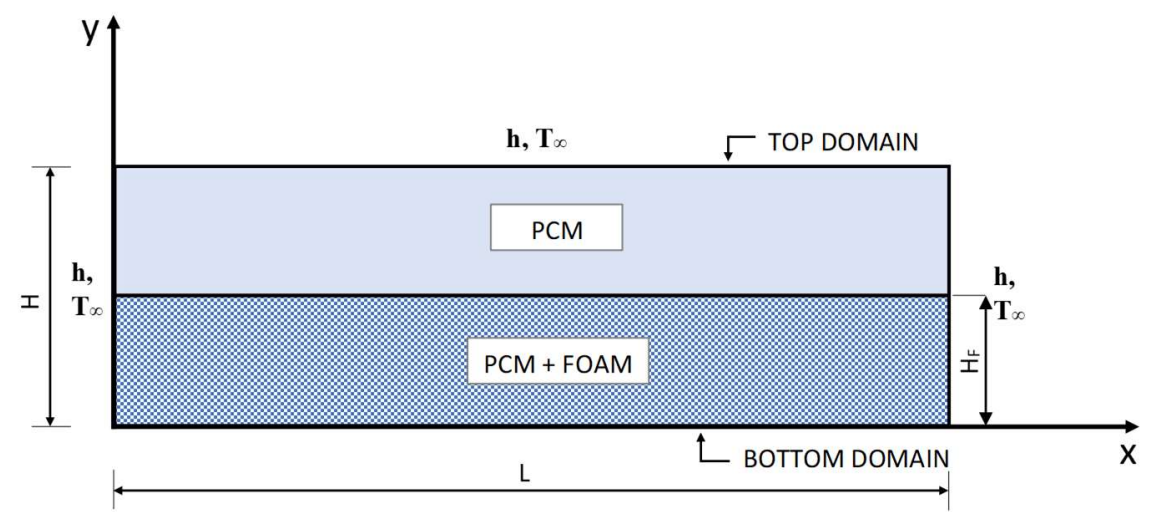

Fig. 1. Physical Domain

The properties of the materials and the copper foam have been reported in Table 1 and Table 2.

Table 1. Properties of materials.

\begin{tabular}{lcll}
\hline Thermal Properties & $\begin{array}{l}\text { Paraffin RT42 } \\
{[10]}\end{array}$ & Copper & Air \\
\hline Density $\left[\mathrm{kg} / \mathrm{m}^{3}\right]$ & 820 & 8978 & 1.225 \\
Specific Heat $[\mathrm{J} / \mathrm{kg} \mathrm{K}]$ & 2000 & 381 & 1006.4 \\
Thermal Conductivity $[\mathrm{W} / \mathrm{m} \mathrm{K}]$ & 0.20 & 387.6 & 0.0242 \\
Dynamic Viscosity $[\mathrm{kg} / \mathrm{m} \mathrm{s}]$ & 0.020 & - & $1.789 \cdot 10^{-5}$ \\
Thermal expansion coefficient $[1 / \mathrm{K}]$ & 0.0001 & - & 0.00333 \\
Melting Heat [J / kg] & 165000 & - & - \\
Solidus Temperature $[\mathrm{K}]$ & 311.15 & - & - \\
Liquidus Temperature $[\mathrm{K}]$ & 316.15 & - & - \\
\hline
\end{tabular}

The enthalpy-porosity method [13] is used to describe the melting phenomenon of PCM, where the solid-liquid interface is not drawn explicitly but the method defines a mixed solidliquid region that represents the "pseudo" porous zone that is the liquid fraction. The liquid fraction $\beta$ has a value of 0 when the zone is totally solid, 1 when it is totally liquid, and between 0 and 1 for the mixed region.

$$
\left\{\begin{array}{lll}
\beta=0 & \text { for } & T<T_{\text {solidus }} \\
\beta=\frac{T-T_{\text {solidus }}}{T_{\text {liquidus }}-T_{\text {solidus }}} & \text { for } & T_{\text {solidus }}<T<T_{\text {liquidus }} \\
\beta=1 & \text { for } & T>T_{\text {liquidus }}
\end{array}\right.
$$


Table 2. Properties of copper foams [12].

\begin{tabular}{lc}
\hline & Cu-40-6.6 \\
\hline Number of pores per inch, PPI & 40 \\
Relative density, $\rho_{R}(\%)$ & 6.6 \\
Porosity, $\varepsilon(-)$ & 0.934 \\
Fiber thickness, $t(\mathrm{~mm})$ & 0.262 \\
Fiber lenght, $l(\mathrm{~mm})$ & 1.109 \\
Surface are per unit of volume, $a_{s v}\left(\mathrm{~m}^{2} \mathrm{~m}^{3}\right)$ & 1635 \\
Permeability, $K\left(\mathrm{~m}^{2}\right)$ & 0.44 \\
Inertia coefficient,$f$ & 0.060 \\
Drag coefficient, $C\left(\mathrm{~m}^{-1}\right)$ & 286
\end{tabular}

In Eq. (1) $\mathrm{T}$ is the local temperature of the cell, $\mathrm{T}_{\text {liquidus }}$ is the upper temperature at which the domain is totally liquid, and $\mathrm{T}_{\text {solidus }}$ is the temperature below which it is totally solid. The solid part of the mixed zone is modeled by adding a source term in the momentum equation. The metal foam is modeled with the Darcy-Forchheimer extended Brinkman model because it behaves as a porous medium; The gravitational acceleration is along the $y$-axis and the Boussinesq approximation is considered to account for the buoyancy force due to natural convection. The thermal interaction between the metal foam and the flows is modeled with the Local Thermal Equilibrium (LTE) assumption, where a unique local temperature function is defined between the porous medium and the fluid flow. The governing equations are as follows:

$$
\begin{gathered}
\frac{\partial u_{p c m}}{\partial x}+\frac{\partial v_{p c m}}{\partial y}=0 \\
\frac{\rho_{p c m}}{\varepsilon}\left(\frac{\partial u_{p c m}}{\partial t}+\frac{u_{p c m}}{\varepsilon} \frac{\partial u_{p c m}}{\partial x}+\frac{v_{p c m}}{\varepsilon} \frac{\partial u_{p c m}}{\partial y}\right)=-\frac{\partial p_{p c m}}{\partial x}+\frac{\mu_{p c m}}{\varepsilon}\left(\frac{\partial^{2} u_{p c m}}{\partial x^{2}}+\frac{\partial^{2} u_{p c m}}{\partial y^{2}}\right)+S_{x} \\
\frac{\rho_{p c m}}{\varepsilon}\left(\frac{\partial v_{p c m}}{\partial t}+\frac{u_{p c m}}{\varepsilon} \frac{\partial v_{p c m}}{\partial x}+\frac{v_{p c m}}{\varepsilon} \frac{\partial v_{p c m}}{\partial y}\right)=-\frac{\partial p_{p c m}}{\partial y}+\frac{\mu_{p c m}}{\varepsilon}\left(\frac{\partial^{2} v_{p c m}}{\partial x^{2}}+\frac{\partial^{2} v_{p c m}}{\partial y^{2}}\right)+S_{y} \\
{\left[(1-\varepsilon)(\rho c)_{m f}+\varepsilon(\rho c)_{p c m}\right]\left(\frac{\partial T_{p c m}}{\partial t}+u_{p c m} \frac{\partial T_{p c m}}{\partial x}+v_{p c m} \frac{\partial T_{p c m}}{\partial y}\right)=} \\
=k_{e f f \mid p c m}\left(\frac{\partial^{2} T_{p c m}}{\partial x^{2}}+\frac{\partial^{2} T_{p c m}}{\partial y^{2}}\right)-\varepsilon \rho_{p c m} H_{L} \frac{\partial \beta}{\partial t}
\end{gathered}
$$

The heat generation rate simulates a typical battery dissipated power of $5.3 \mathrm{~kW}$.

In the equations, $\rho$ and $\mathrm{c}$ are the density and specific heat, respectively; $\mathrm{u}$ and $\mathrm{v}$ are the velocity in the $\mathrm{x}$ and $\mathrm{y}$ directions, respectively; $\varepsilon$ is the porosity of the metal foam; $\mathrm{p}$ is the relative pressure; $\mu$ is the dynamic viscosity of the PCM or air; $\mathrm{S}$ is the source term; $\mathrm{V}$ is the velocity vector of the PCM in the liquid or air phase; and et is the time. The subscripts Lit, 
pcm, and air referred to the battery, phase change material, and air, respectively. Regarding the local temperature, the LTE approach is used and thus the temperature foam in the PCM domain is equal to the PCM temperature and the same assumption is set in the air domain.

The source terms in the PCM momentum equations are:

$$
\begin{gathered}
S_{x}=\left(\frac{(1-\beta)^{2}}{\left(\beta^{3}+0.001\right)^{3}} A_{m u s h}+\frac{\mu_{p c m}}{K}+\frac{C_{F}}{\sqrt{K}} \rho_{p c m}\left|\vec{V}_{p c m}\right|\right) v_{p c m} \\
S_{y}=\left(\frac{(1-\beta)^{2}}{\left(\beta^{3}+0.001\right)^{3}} A_{m u s h}+\frac{\mu_{p c m}}{K}+\frac{C_{F}}{\sqrt{K}} \rho_{p c m}\left|\vec{V}_{p c m}\right|\right) v_{p c m}+\rho_{p c m} g \gamma_{p c m}\left(T_{p c m}-T_{0}\right)
\end{gathered}
$$

The first term is the Kozeny - Carman term that models the presence of the solid part of the PCM during melting. $A_{\text {mush }}$ is the mushy zone constant that takes into account the velocity damping during solidification. Its value is fixed at $10^{5} \mathrm{~kg} /\left(\mathrm{m}^{3} \mathrm{~s}\right)$. The second term is the Darcy term where $K$ is the permeability of the porous medium and the third term is the Forchheimer term, where $\mathrm{C}_{\mathrm{F}}$ is the inertial strength factor.

The details of equation 7 can be found in [14].

Permeability and resistance coefficients are calculated from:

$$
\left\{\begin{array}{l}
K=0.00073(1-\varepsilon)^{-0.224}\left(\frac{d_{f}}{d_{p}}\right)^{-1.11} d_{p}^{2} \\
C_{F}=0.00212(1-\varepsilon)^{-0.132}\left(\frac{d_{f}}{d_{p}}\right)^{-1.63} \\
\frac{d_{f}}{d_{p}}=1.18 \sqrt{\frac{1-\varepsilon}{3 \pi}}\left(\frac{1}{1-e^{1(1-\varepsilon) / 0.04}}\right) \\
d_{p}=\frac{0.0224}{\omega}
\end{array}\right.
$$

The last term is the Boussinesq approximation where $\mathrm{g}$ is the acceleration modulus of gravity, $\gamma_{\mathrm{pcm}}$ is the thermal expansion coefficient of the PCM, and $T_{0}$ is the operating temperature, set to $310 \mathrm{~K} . k_{\text {eff }}$ is the effective thermal conductivity calculated by [15]:

$$
\left\{\begin{array}{l}
k_{e f f \mid p c m}=(1-\varepsilon) k_{m f}+\varepsilon k_{p c m} \\
k_{e f f \mid a i r}=(1-\varepsilon) k_{m f}+\varepsilon k_{\text {air }}
\end{array}\right.
$$

The boundary conditions have been reported in Table 3 .

\section{Numerical model}

ANSYS Fluent [16] was used to solve the governing equations using the finite volume method. A transient mode with a time interval of $1 \mathrm{~s}$ was used. The phenomenon related to coupled velocity-pressure fields has been solved using the SIMPLE algorithm developed by Patankar [17]. A second order upwind model is used to treat the convective terms for the 
1/2 (20 mm of foam with PCM and the remaining $20 \mathrm{~mm}$ of PCM only) and partially filled configuration $3 / 4$ (30 $\mathrm{mm}$ of foam with PCM and the remaining $10 \mathrm{~mm}$ of PCM only).

These materials were analysed both individually and compared to each other to understand the differences. Analyses were carried out in adiabatic conditions, $\mathrm{h}=0$, in natural convection, $\mathrm{h}=20 \mathrm{~W} / \mathrm{m}^{2} \mathrm{~K}$, and for forced convection, $\mathrm{h}=200 \mathrm{~W} / \mathrm{m}^{2} \mathrm{~K}$. Results are given in terms of liquid fraction, Figure 2, and average temperatures of both layers Top Domain and Bottom Domain, Figure 3.

Simulations obtained with PCM-filled copper foam showed that the mean temperatures between the upper domain and lower domain parts were closest. Of these, fully filled simulations have the lowest average temperatures compared to simulations conducted with combinations of copper foams and partially filled PCM. This phenomenon visible in Figure 2 can be explained by the fact that when the battery temperature approaches the critical temperature, the PCM begins to melt and the foam uniforms the phenomenon along the entire domain in which it is introduced. Therefore, the temperature tends to remain on levels more suited to the thermal safety values of the battery.

In Figure 3 it is possible to see that, as regards the simulations obtained with PCM filled with copper foam and with heat transfer coefficient $h=0\left[\mathrm{~W} / \mathrm{m}^{2} \mathrm{~K}\right]$, the average temperatures of the Top (Top Domain) and Bottom layers were close, despite having the highest average temperatures and that, as the heat transfer coefficient $\mathrm{h}=20,200\left[\mathrm{~W} / \mathrm{m}^{2} \mathrm{~K}\right]$ increases, the average temperatures drop considerably due to the onset of convection. The greatest difference between the average temperatures of the Top and Bottom Domains were obtained with simulations that involved the use of PCM only. In fact, the average temperature measured on the Bottom layer of the domain (which receives the heat flux), turns out to be at very high average temperatures, which make the PCM completely liquefy, thus making it dangerous for the thermal safety parameters of lithium batteries. Zones instead above the Bottom layer up to the Top Domain part are at extremely lower average temperatures, not triggering the transition between solid and liquid and therefore presenting paraffin in the solid state.

\section{Conclusions}

In this research work, an electric vehicle battery cooler was implemented by numerically realizing a 2D domain. The materials used for this purpose were 40 PPI copper foams and a PCM of paraffin. The temperatures and melting times of the PCM have been reported as time changes and various types of partially filled analysed.

From the results obtained, it can be seen that the average temperatures obtained with only PCM show extremely different average temperatures between Top domain and Bottom. In fact, while the Bottom area (the layer between the battery and the PCM) turns out to be at extremely high average temperatures with liquefied PCM, the areas above Bottom are instead at much lower average temperatures. In fact, comparing the Bottom layer of only PCM with PCM totally filled with copper foam, we have that at $\mathrm{h}=0$ the PCM alone is at average temperatures $8.14 \%$ higher than the PCM totally filled with copper foam. This difference, in terms of average temperature, increases with $\mathrm{h}=20 \mathrm{~W} / \mathrm{m}^{2} \mathrm{~K}$ reaching $9.49 \%$ and even more with $\mathrm{h}=200 \mathrm{~W} / \mathrm{m}^{2} \mathrm{~K}$ reaching $17.56 \%$. 


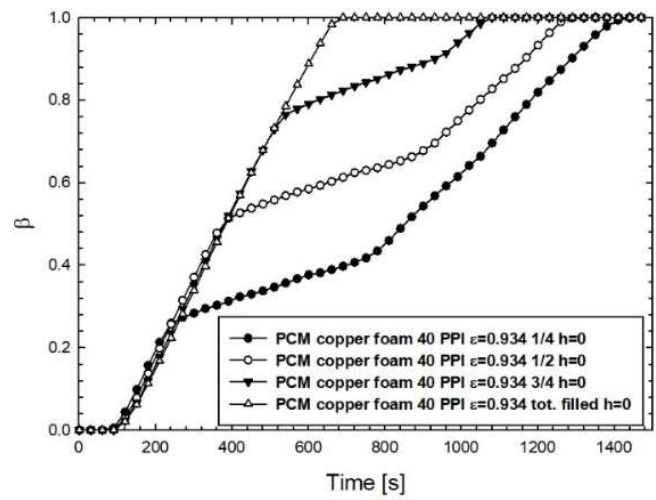

(a)

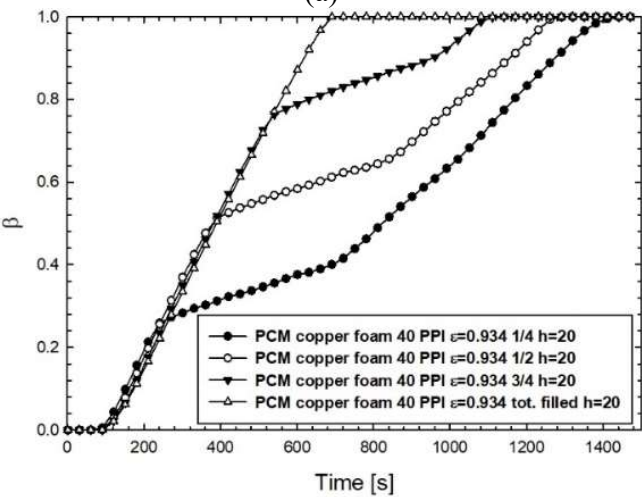

(b)

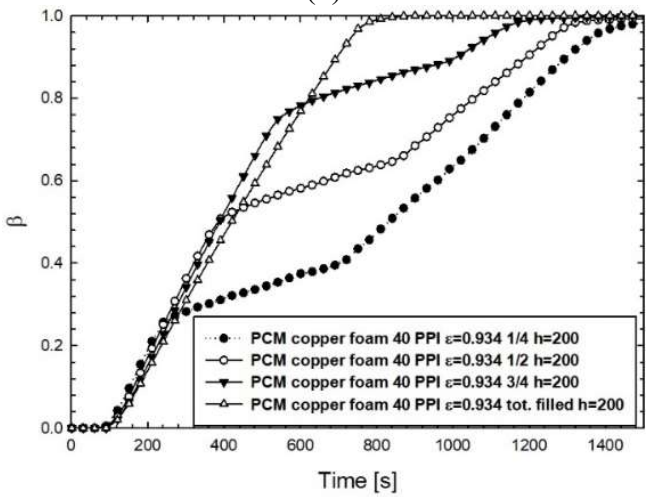

(c)

Fig.2. Liquid fraction of domain for partially and total filled 40 PPI copper foam filled by PCM $h=0$ (a), 20 (b), 200 (c) $\left[\mathrm{W} / \mathrm{m}^{2} \mathrm{~K}\right]$.

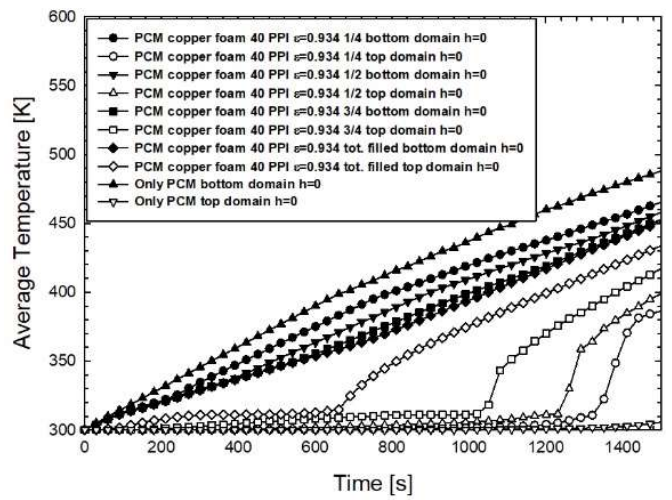

(a)

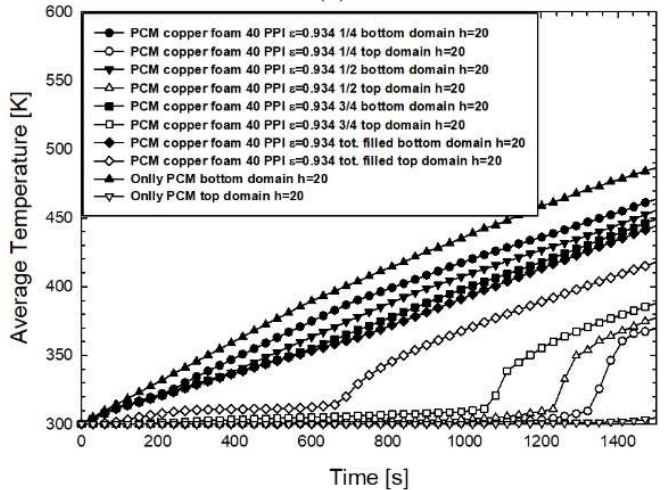

(b)

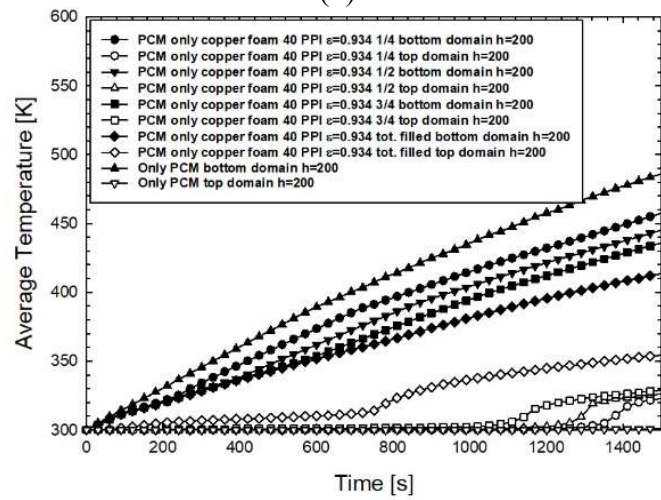

(c)

Fig. 3. Average temperatures of Bottom and Top domain for only PCM and 40 PPI copper foam filled PCM (a) h=0 [W/m² K], (b) h=20 [W/m $\left./ \mathrm{m}^{2} \mathrm{~K}\right], \mathrm{h}=100$ $\left[\mathrm{W} / \mathrm{m}^{2} \mathrm{~K}\right]$.

This appears to be out of line with the safety parameters of the battery. The cases that have been analysed with the combination of copper foams with PCM appear to have the average temperatures of the two layers (Top domain and Bottom) closer and lower than the only PCM case. In particular, the closest average temperatures between the Top domain and Bottom layer were obtained with the totally filled (totally filled with copper foams and PCM) and this makes them more in tune with the optimal operating parameters of lithium batteries. 
Results show that the combination of phase change materials with metal foams is advantageous for the thermal control of lithium batteries.

\section{Nomenclature}

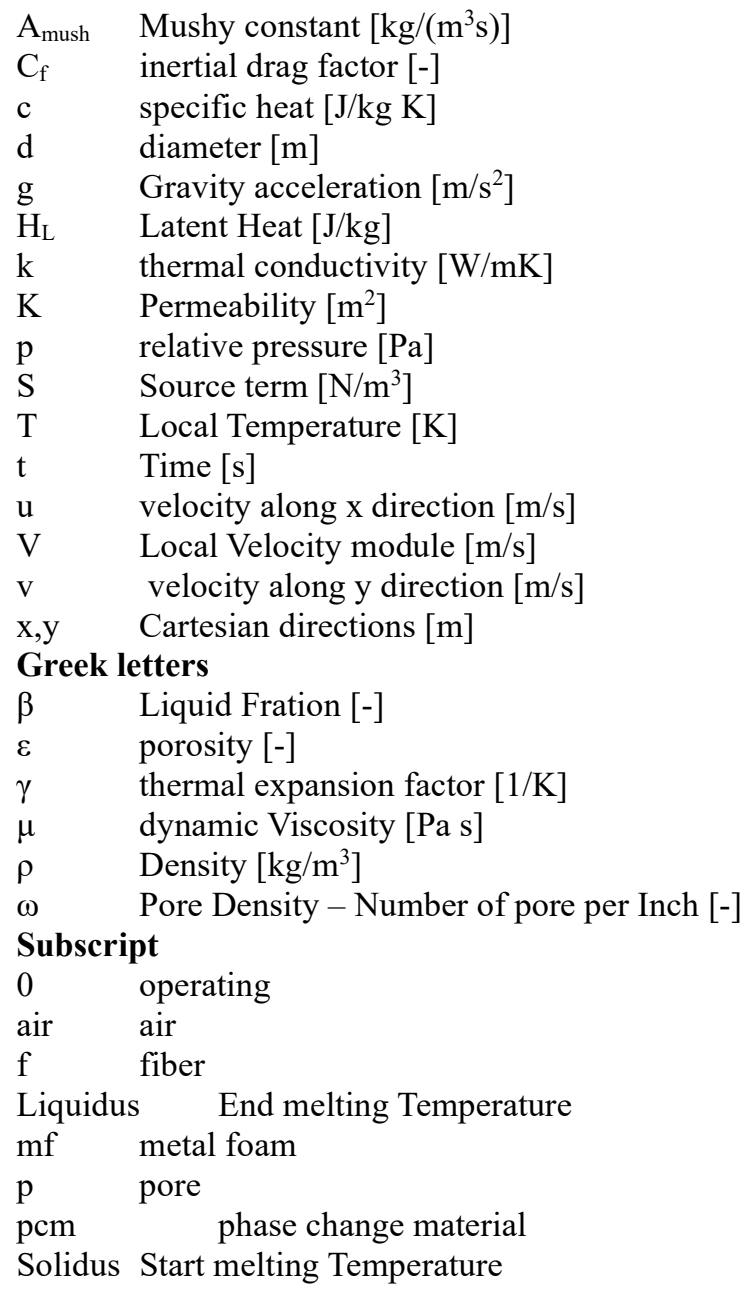

\section{Acknowledgements}

This research was partially funded by MIUR (Ministero dell'Istruzione, dell'Universit a e della Ricerca), grant number PRIN- 2017F7KZWS and by Università degli Studi della Campania "Luigi Vanvitelli" with the grant number D.R. n. 138 under NanoTES project V:ALERE program 2020.

\section{References}

1. W. Libeer, F. Ramos, C. Newton, M. Alipanahrostami, C. Depcik, X. Li, Int. J. Heat Mass Transf. 100, 215-223 (2016).

2. S. Landini, J. Leworthy, T.S. O’Donovan, J. of Energy Storage, 25 (2018). 
3. K. Lafdi, O. Mesalhy, S. Shaikh, J. of Applied Physics, 102 (2007).

4. M. Safdari, R. Ahmadi, S. Sadeghzadeh, Energy, 193 (2020)

5. B. Buonomo, D. Ercole, O. Manca, F. Menale, Energy Procedia, 148, 1175-1182 (2018).

6. B. Buonomo, O. Manca, F. Menale, S. Nardini, J. Phys.: Conf. Ser, 1868012015 (2021).

7. M. Mashayekhi, E. Houshfar, M. Ashjaee, Applied Thermal Engineering, 178 (2020).

8. A. Veismoradi, A. Modir, M Ghalambaz, A. Chamkha, Int. Jour. of T. Sci. 157 (2020).

9. M. M. El Idi, M. Karkri, M Kraiem, J. of Energy Storage, 33 (2021).

10. Rubitherm GmBH, www.rubitherm.de

11. Sparkfan electronic, Lithium Ion Rechargeable Batteries Technical Handbook, [online] https://cdn.sparkfun.com/datasheets/Prototyping/Lithium\%20Ion\%20Battery\%20MSD $\underline{\text { S.pdf }}$

12. S.Mancin, C. Zilio, A. Diani, L. Rossetto, Exp. T. and Fluid Science, 36, 224-232 (2012).

13. A. A. Al-abidi, S. B. Mat, K. Sopian, M. Y. Sulaiman, A. T. Mohammed, Ren. and Sustainable Energy Reviews, 20, 353-363 (2013).

14. V.V. Calmidi, R.L. Mahajan, J. Heat Transfer, 122, 557-565 (2000).

15. Z. Liu, Y. Yao, H. Wu, Applied Energy, 112, 1222-1232 (2013).

16. Ansys Fluent 18.0, Ansys Inc

17. S.V. Patankar, Hemisphere Publishing Corporation. New York, 1980. 